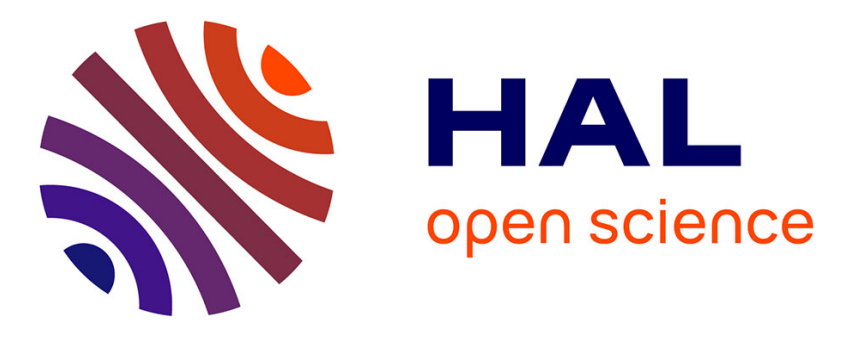

\title{
Stability of homogeneous nonlinear systems with sampled-data inputs
}

\author{
Emmanuel Bernuau, Emmanuel Moulay, Patrick Coirault
}

\section{To cite this version:}

Emmanuel Bernuau, Emmanuel Moulay, Patrick Coirault. Stability of homogeneous nonlinear systems with sampled-data inputs. Automatica, 2017, 85, pp.349 - 355. 10.1016/j.automatica.2017.07.048 . hal-01724037

\section{HAL Id: hal-01724037 \\ https://hal.science/hal-01724037}

Submitted on 23 Dec 2020

HAL is a multi-disciplinary open access archive for the deposit and dissemination of scientific research documents, whether they are published or not. The documents may come from teaching and research institutions in France or abroad, or from public or private research centers.
L'archive ouverte pluridisciplinaire HAL, est destinée au dépôt et à la diffusion de documents scientifiques de niveau recherche, publiés ou non, émanant des établissements d'enseignement et de recherche français ou étrangers, des laboratoires publics ou privés. 


\title{
Stability of homogeneous nonlinear systems with sampled-data inputs *
}

\author{
Emmanuel Bernuau $^{\mathrm{a}}$, Emmanuel Moulay $^{\mathrm{b}}$, Patrick Coirault ${ }^{\mathrm{c}}$ \\ ${ }^{\mathrm{a}}$ GENIAL (UMR INRA 1145), AgroParisTech, 1 Avenue des Olympiades, 91744 Massy Cedex, France \\ ${ }^{\mathrm{b}}$ XLIM (UMR CNRS 7252), Université de Poitiers, 11 bd Marie et Pierre Curie, 86962 Futuroscope Chasseneuil Cedex, France \\ ${ }^{\mathrm{c}}$ LIAS (EA 6315), Université de Poitiers, 2 rue Pierre Brousse, 86073 Poitiers Cedex 9, France
}

\begin{abstract}
The main goal of this article is to use properties of homogeneous systems for addressing the problem of stability for a class of nonlinear systems with sampled-data inputs. This nonlinear strategy leads to several kinds of stability, i.e. local asymptotic stability, global asymptotic stability or global asymptotic set stability, depending on the sign of the degree of homogeneity. The results are illustrated with the case of the double integrator.
\end{abstract}

Key words: Sampled-data systems, Nonlinear systems, Homogeneity, Lyapunov stability, Lyapunov functions.

\section{Introduction}

Feedback control systems wherein the control loops are closed through a real-time network are called Networked Control Systems (NCSs) [1]. In NCSs, the presence of packet-based communication, network delays, limited bandwidth and packet dropouts is inevitable. NCSs are widely studied in automatic control since several years $[2,3]$.

When studying NCSs, a crucial problem is to determine whether some stability properties pertain through sampled-data inputs [4]. Moreover, due to, for instance, data packet dropouts [5], the inputs are not only sampled but also usually nonuniformly sampled with respect to time and this is called aperiodic sampled-data inputs [6]. Several approaches have been developed to solve the problem of stability for systems with sampled-data inputs, such as the input/output approach $[7,8]$ or the sum of squares approach [9]. Moreover, different dynamical models as hybrid systems $[1,10]$, discrete-time systems

\footnotetext{
* This paper was not presented at any IFAC meeting. Corresponding author E. Bernuau. Tel. +33 (0)1 699350 83; Fax +33 (0)169935020.

Email addresses: emmanuel. bernuau@agroparistech.fr (Emmanuel Bernuau),

emmanuel.moulay@univ-poitiers.fr (Emmanuel Moulay), patrick.coirault@univ-poitiers.fr (Patrick Coirault).
}

[11] or time-delay systems $[5,12-14]$ have also been used to tackle this problem. Most of these strategies [7-9] are developed under the emulation method where the controller is first designed in continuous time and then implemented as a sampled-data controller $[2,15]$.

The notion of homogeneity was introduced in [16] and [17] and developed by many authors, for instance in [18-20]. In [21], a new nonlinear approach based on the input-to-state stability (ISS) properties of homogeneous systems has been introduced for studying self-triggered control for nonlinear systems. We know from [22,23] that the emulation of a feedback law, which globally asymptotically stabilizes the origin of a nonlinear continuoustime system, leads in general to semi-global practical stability in the sampled-data context. In our article, we show that if the nonlinear system satisfies in addition a homogeneity property, then the global asymptotic stability, local asymptotic stability or global asymptotic set stability are preserved under emulation depending on the degree of homogeneity.

The main result shows that if it is possible to build a stabilizing static feedback control for a continuous system such that the closed-loop system with sampled-data inputs satisfies a homogeneity property, then it is possible to preserve different notions of stability that depend on the degree of homogeneity $\kappa$ for the closed-loop system with aperiodic sampled-data inputs. When $\kappa=0$, 
the closed-loop system is globally asymptotically stable if the maximum sampling period $h$ is shorter than a constant $H$. Linear systems are special cases of homogeneous systems of degree 0 and our result hence shows that homogeneity explains this feature of linear systems. We refer to $[1,14,24]$ for different strategies used in the literature for estimating $H$ in the linear case. When $\kappa>0$, the closed-loop system achieves local asymptotic stability, while when $\kappa<0$, it achieves global asymptotic set stability. In these two cases, the results remain true regardless of $h<+\infty$, although the size of the domain of attraction and of the limit set do depend on $h$. With the use of an homogeneous observer, the results are then extended to continuous systems with an output. The results are finally applied to the case of the double integrator which is rather important in control theory despite its simplicity $[25,26]$.

The article is organized as follows. After some notations and definitions given in Section 2, we develop the main results of the article in Section 3. The example of the double integrator is treated in Section 4. Finally, a conclusion is addressed in Section 5.

\section{Notations and definitions}

Let us introduce the following notations:

- $\mathbb{R}_{+}=\{x \in \mathbb{R}: x \geq 0\}$, where $\mathbb{R}$ is the set of real numbers.

- $|\cdot|$ denotes the absolute value in $\mathbb{R},\|\cdot\|$ denotes the Euclidean norm on $\mathbb{R}^{n}$.

- For $r_{1}, r_{2}, \ldots, r_{n}, \operatorname{Diag}\left(r_{1}, \ldots, r_{n}\right)$ denotes the diagonal matrix of dimension $n \times n$ with $k^{\text {th }}$ diagonal entry $r_{k}$.

- A measurable function $d: \mathbb{R}_{+} \rightarrow \mathbb{R}^{m}$ is locally essentially bounded if for any $0 \leq a<b$, the function $d$ is essentially bounded on the segment $[a, b] . \mathcal{L}_{\text {loc }}^{\infty}$ denotes the set of locally essentially bounded functions $d: \mathbb{R}_{+} \rightarrow \mathbb{R}^{m}$.

- A continuous function $\alpha: \mathbb{R}_{+} \rightarrow \mathbb{R}_{+}$belongs to the class $\mathcal{K}$ if $\alpha(0)=0$ and the function is strictly increasing. A function $\alpha: \mathbb{R}_{+} \rightarrow \mathbb{R}_{+}$belongs to the class $\mathcal{K}_{\infty}$ if $\alpha \in \mathcal{K}$ and it is unbounded.

- A continuous function $\beta: \mathbb{R}_{+} \times \mathbb{R}_{+} \rightarrow \mathbb{R}_{+}$belongs to the class $\mathcal{K} \mathcal{L}$ if $\beta(\cdot, t) \in \mathcal{K}_{\infty}$ for each fixed $t \in \mathbb{R}_{+}$ and if for each fixed $s \in \mathbb{R}_{+}$the function $t \mapsto \beta(s, t)$ is decreasing to 0 .

- The notation $d_{x} V$ (resp. $\left.d_{x} \Phi\right)$ stands for the differential of the function $V$ (resp. the diffeomorphism $\Phi$ ) at the point $x$.

- $\left\lfloor\left. x\right|^{\alpha}=|x|^{\alpha} \operatorname{sign}(x)\right.$ where $x \in \mathbb{R}$ and $\alpha>0$.

- Given that we will deal with a variety of suprema, we will use a compact notation in the computations. For instance, the notation $\sup \{f(x, y): g(x)=0, h(y) \leq$ $1\}$ stands for $\sup _{x \in E, y \in F} f(x, y)$ where $E=\left\{x \in \mathbb{R}^{n}\right.$ : $g(x)=0\}$ and $F=\left\{y \in \mathbb{R}^{n}: h(y) \leq 1\right\}$.
Consider the following system with $f$ continuous

$$
\dot{x}=f(x), \quad x \in \mathbb{R}^{n} .
$$

Let us recall the definitions of Lyapunov set stability given for instance in [27].

Definition $1 A$ compact set $K \subset \mathbb{R}^{n}$ is:

- stable w.r.t. the system (1) if for any neighborhood $U$ of $K$, there exists a neighborhood $W$ of $K$ such that for any maximal solution $x(t)$ of $(1)$, if there exists $t_{0}$ such that $x\left(t_{0}\right) \in W$, then $x(t)$ is defined for all $t \geq t_{0}$ and $x(t) \in U$ for all $t \geq t_{0}$;

- locally attractive w.r.t. the system (1) if there exists a neighborhood $U$ of $K$ such that for any maximal solution $x(t)$ of $(1)$, if there exists $t_{0}$ such that $x\left(t_{0}\right) \in U$, then $x(t)$ is defined for all $t \geq t_{0}$ and $x(t) \rightarrow K$ when $t \rightarrow+\infty$ (the domain of attraction of a locally attractive set is the biggest set $U$ for which the preceding point hold);

- globally attractive w.r.t. the system (1) if it is locally attractive and if its domain of attraction is $\mathbb{R}^{n}$;

- locally (resp. globally) asymptotically stable w.r.t. the system (1) if it is stable and locally (resp. globally) attractive w.r.t. the system (1);

- unstable if it is not stable.

Let us consider the following nonlinear system

$$
\dot{x}=f(x, \Delta)
$$

where $x \in \mathbb{R}^{n}$ is the state, $\Delta \in \mathcal{L}_{\text {loc }}^{\infty}$ is the external input and $f: \mathbb{R}^{n} \times \mathbb{R}^{m} \rightarrow \mathbb{R}^{n}$ is continuous. Let us recall the definition of input-to-state stability given for instance in [28].

Definition 2 The system (2) is called input-to-state stable (ISS) if there exist some functions $\beta \in \mathcal{K} \mathcal{L}$ and $\gamma \in \mathcal{K}$ such that for any input $\Delta \in \mathcal{L}_{\text {loc }}^{\infty}$ and any $x_{0} \in \mathbb{R}^{n}$ it holds that

$$
\|x(t)\| \leq \beta(\|x(0)\|, t)+\underset{\tau \in[0, t]}{\operatorname{ess} \sup } \gamma(\|\Delta(\tau)\|) \quad \forall t \geq 0
$$

where $x(t)$ is the solution of the system (2) satisfying $x(0)=x_{0}$. The function $\gamma$ is called a nonlinear asymptotic gain.

The most common notion of homogeneity is the weighted homogeneity, based on a particular choice of the coordinates, while the most generic one is the geometric homogeneity, which is coordinate free $[18,19]$. We use in the sequel the framework of geometric homogeneity.

Definition 3 A vector field $\nu$ on $\mathbb{R}^{n}$ is called an Euler vector field if $\nu$ is of class $C^{1}$, complete (i.e. the maximal solutions of $\dot{x}=\nu(x)$ are defined on $\mathbb{R}$ ) and if the origin is a globally asymptotically equilibrium of $-\nu$. 
Definition 4 Let $\nu$ be an Euler vector field on $\mathbb{R}^{n}, \Phi^{s}(x)$ denotes the value of the flow ${ }^{1}$ of $\nu$ at time $s$ with initial condition $x$. A function $V: \mathbb{R}^{n} \rightarrow \mathbb{R}$ is $\nu$-homogeneous of degree $\kappa \in \mathbb{R}$ if for all $x \in \mathbb{R}^{n}$ and all $s \in \mathbb{R}$ we have $V\left(\Phi^{s}(x)\right)=e^{\kappa s} V(x)$. A vector field $f$ on $\mathbb{R}^{n}$ is $\nu$ homogeneous of degree $\kappa \in \mathbb{R}$ if for all $x \in \mathbb{R}^{n}$ and all $s \in \mathbb{R}$ we have $f\left(\Phi^{s}(x)\right)=e^{\kappa s} d_{x} \Phi^{s} f(x)$.

A direct application of the chain rule then gives us the following result.

Proposition 5 Let $\nu$ be an Euler vector field on $\mathbb{R}^{n}$. If $V: \mathbb{R}^{n} \rightarrow \mathbb{R}$ is differentiable and $\nu$-homogeneous of degree $\kappa$, then for all $x \in \mathbb{R}^{n}$ and all $s \in \mathbb{R}$

$$
d_{\Phi^{s}(x)} V \cdot d_{x} \Phi^{s}=e^{\kappa s} d_{x} V .
$$

Remark 6 If we consider a matrix $A \in \mathbb{R}^{n \times n}$ such that $-A$ is Hurwitz then the vector field defined by $\nu(x)=A x$ is an Euler vector field and the flow of $\nu$ verifies $\Phi^{s}(x)=$ $\exp (A s) x$. In particular, if $A=\operatorname{Diag}\left(r_{1}, \ldots, r_{n}\right)$ with $r_{1}, \ldots, r_{n}>0$, the vector field $\nu(x)=A x$ is Euler and we find $\Phi^{s}(x)=\operatorname{Diag}\left(e^{r_{1} s}, \ldots, e^{r_{n} s}\right) x$. The homogeneity defined by such an Euler vector field is usually referred to as weighted homogeneity, the coefficients $r_{1}, \ldots, r_{n}$ are called the weights and $\mathbf{r}=\left[r_{1}, \ldots, r_{n}\right]$ is called the generalized weight [30]. Let us finally mention that homogeneity with respect to an Euler vector field defined by a generalized weight $\mathbf{r}$ is usually simply referred to as r-homogeneity.

Definition 7 Let $\nu$ be an Euler vector field on $\mathbb{R}^{n}$. A $\nu$ homogeneous norm is a function $N: \mathbb{R}^{n} \rightarrow \mathbb{R}$ such that:

(1) $N$ is positive definite;

(2) $N$ is $\nu$-homogeneous of degree 1 ;

(3) $N$ is continuous.

Example 8 Let $\mathbf{r}=\left[r_{1}, \ldots, r_{n}\right]$ be a generalized weight. For any $\rho>0$, a $\mathbf{r}$-homogeneous norm is defined by $N_{\rho}(x)=\left(\sum_{i=1}^{n}\left|x_{i}\right|^{\frac{\rho}{r_{i}}}\right)^{\frac{1}{\rho}}$. Furthermore, $N_{\infty}(x)=\sup _{i}\left|x_{i}\right|^{\frac{1}{r_{i}}}$ also defines a $\mathbf{r}$-homogeneous norm.

Remark 9 A homogeneous norm is always radially unbounded, following [18, Lemma 4.1]

The following proposition is a direct consequence of [18, Lemma 4.2].

Proposition 10 Let $N_{1}$ and $N_{2}$ be two $\nu$-homogeneous norms. Then there exist $a, b>0$ such that, for all $x \in \mathbb{R}^{n}$ :

$$
a N_{1}(x) \leq N_{2}(x) \leq b N_{1}(x)
$$

\footnotetext{
1 See [29], Chapter 6 for details.
}

\section{Main results}

Let us consider the following nonlinear system

$$
\dot{x}=F(x, u)
$$

defined by a continuous function $F: \mathbb{R}^{n} \times \mathbb{R}^{m} \rightarrow \mathbb{R}^{n}$ and assume known a continuous static feedback $u: \mathbb{R}^{n} \rightarrow \mathbb{R}^{m}$ such that the origin is a globally asymptotically stable equilibrium of the closed-loop system $\dot{x}=F(x, u(x))$. Remark that, in this formulation, the control $u$ may depend on the state $x$ through a static output feedback $u(x)=v(y)$, where $y=g(x)$ is an output. Since in networked communication, the state information is only updated at discrete time instants, we attempt at giving conditions based on homogeneity under which some stability properties pertain for the system (3). More precisely, we consider a maximum sampling period $0<h<+\infty$, a minimum sampling period $\eta>0$ and a sequence of sampling times $\left(t_{k}\right)_{k \in \mathbb{N}}$ such that $t_{0}=0$ and

$$
\eta \leq t_{k+1}-t_{k} \leq h
$$

Due to the sampling, the control is now $u(t)=u\left(x\left(t_{k}\right)\right)$ for all $t \in\left[t_{k}, t_{k+1}\right.$ ) (sample and hold). The system can therefore be rewritten under the following form

$$
\dot{x}(t)=F\left(x(t), u\left(x\left(t_{k}\right)\right)\right), \quad t \in\left[t_{k}, t_{k+1}\right) .
$$

We consider the following assumption on system (5).

Assumption 11 There exists a matrix $A \in \mathbb{R}^{n \times n}$ such that $-A$ is Hurwitz and a degree $\kappa \in \mathbb{R}$ such that

$$
F(\exp (A s) x, u(\exp (A s) z))=e^{\kappa s} \exp (A s) F(x, u(z))
$$

for all $x, z \in \mathbb{R}^{n}$ and all $s \in \mathbb{R}$.

Assumption 11 is a homogeneity property of system (5). Indeed, if we consider the vector field $\bar{F}$ defined on $\mathbb{R}^{n} \times \mathbb{R}^{n}$ by $\bar{F}(x, z)=(F(x, u(z)), 0)^{T}$, then Assumption 11 is equivalent to the $\nu$-homogeneity of $\bar{F}$ with $\nu(x, z)=(A x, A z)^{T}$. In particular, this assumption implies that $F(x, u(x))$ is homogeneous. A class of systems that satisfy Assumption 11 is, for example, the chain of integrators and its nonlinear extension studied in [31]. Let us now give the main result of the article.

Theorem 12 Assume that the sampled system (5) is such that the sampling times satisfy (4) and that Assumption 11 holds. Consider $\nu(x)=A x$ and $N$ any $\nu$ homogeneous norm.

(1) If $\kappa>0$ then the origin is a locally asymptotically stable equilibrium of the system (5) and there exists $\xi>0$ such that the set $\left\{x \in \mathbb{R}^{n}: N(x) \leq \xi h^{-1 / \kappa}\right\}$ is a subset of the domain of attraction. 
(2) If $\kappa=0$ then there exists $H>0$ such that if $h<H$ then the origin is a globally asymptotically stable equilibrium of the system (5).

(3) If $\kappa<0$ then there exists $\xi>0$ such that the set $\left\{x \in \mathbb{R}^{n}: N(x) \leq \xi h^{-1 / \kappa}\right\}$ is globally asymptotically stable w.r.t. the system (5).

We will need the following result to prove Theorem 12 .

Lemma 13 [32, Theorem 11] Assume that $f: \mathbb{R}^{n} \times$ $\mathbb{R}^{m} \rightarrow \mathbb{R}^{n}$ is continuous and that there exist $\nu$ an Euler vector field on $\mathbb{R}^{n}$ with flow denoted $\Phi^{s}(x), \tilde{\nu}$ an Euler vector field on $\mathbb{R}^{m}$ with flow denoted $\tilde{\Phi}^{s}(d)$ and $\kappa \in \mathbb{R}$ such that for all $x \in \mathbb{R}^{n}$ and all $z \in \mathbb{R}^{m}$,

$$
f\left(\Phi^{s}(x), \tilde{\Phi}^{s}(z)\right)=e^{\kappa s} d_{x} \Phi^{s} f(x, z) .
$$

Assume also that the origin is a globally asymptotically stable equilibrium of the system $\dot{x}=f(x, 0)$. Then the system (2) is ISS w.r.t. an input $\Delta$. Furthermore, for any $\nu$-homogeneous norm $N$ and any $\tilde{\nu}$-homogeneous norm $\tilde{N}$, there exist $C>0$ such that

$$
N(x(t)) \leq \max \{\beta(N(x(0)), t) ; C \underset{\tau \in[0, t]}{\operatorname{ess} \sup } \tilde{N}(\Delta(\tau))\}
$$

with $\beta \in \mathcal{K} \mathcal{L}$ defined by

$$
\beta(r, t)=\left\{\begin{array}{lr}
r e^{-\frac{a}{2} t} & \kappa=0 \\
\max \left\{\left[r^{-\kappa / \mu}+\frac{a \kappa}{2 \mu} t\right]^{-\mu / \kappa}, 0\right\} & \kappa \neq 0
\end{array}\right.
$$

where $a>0$ and $\mu>\max (-\kappa, 0)$ are positive constants.

Proof of Theorem 12. The proof of the theorem is divided into 6 steps.

I. We show that it is sufficient to prove the result for a given $h$ when $\kappa \neq 0$.

II. We rewrite the system and introduce some notation.

III. We prove a preliminary fact on the increase rate of $N(x(t))$.

IV. We prove the theorem for $\kappa>0$.

$\mathrm{V}$. We prove the theorem for $\kappa=0$.

VI. We prove the theorem for $\kappa<0$.

Step $I$. When $\kappa \neq 0$, assume that the results of Theorem 12 hold for a given maximal sampling period $h_{0}>0$. Let us prove that the results then hold for any $h>0$. Indeed, consider $s \in \mathbb{R}$ such that $h=e^{-\kappa s} h_{0}$. Consider a solution $x(t)$ of $(5)$ with $\left(t_{k}\right)_{k \in \mathbb{N}}$ the sequence of sampling times. Then consider $z(t)=\exp (A s) x\left(e^{\kappa s} t\right)$. If $t_{k} \leq e^{\kappa s} t<t_{k+1}$, we have

$$
\dot{z}(t)=e^{\kappa s} \exp (A s) F\left(x\left(e^{\kappa s} t\right), u\left(x\left(t_{k}\right)\right)\right) .
$$

From Assumption 11, it follows that

$$
\begin{aligned}
\dot{z}(t) & =F\left(\exp (A s) x\left(e^{\kappa s} t\right), u\left(\exp (A s) x\left(e^{\kappa s} e^{-\kappa s} t_{k}\right)\right)\right) \\
& =F\left(z(t), u\left(z\left(e^{-\kappa s} t_{k}\right)\right)\right) .
\end{aligned}
$$

Hence the curve $z(t)=\exp (A s) x\left(e^{\kappa s} t\right)$ is a solution of (5) with sampling times $\left(e^{-\kappa s} t_{k}\right)_{k \in \mathbb{N}}$. It follows that the mapping $x(t) \mapsto \exp (A s) x\left(e^{\kappa s} t\right)$ is a bijection between the solutions of (5) with sampling times $\left(t_{k}\right)_{k \in \mathbb{N}}$ and the solutions of (5) with sampling times $\left(e^{-\kappa s} t_{k}\right)_{k \in \mathbb{N}}$. Now, in the case $\kappa>0$ the local asymptotic stability for the maximal sampling period $h_{0}$ ensures the existence of $\beta_{0} \in \mathcal{K} \mathcal{L}$ such that

$$
N\left(x_{0}\right) \leq \xi h_{0}^{-1 / \kappa} \Rightarrow N(x(t)) \leq \beta_{0}\left(N\left(x_{0}\right), t\right), \quad \forall t \geq 0 .
$$

By the point (2) of Definition $7, N\left(x_{0}\right) \leq \xi h_{0}^{-1 / \kappa}$ is equivalent to $N\left(z_{0}\right) \leq e^{s} \xi h_{0}^{-1 / \kappa}=\xi\left(e^{-\kappa s} h_{0}\right)^{-1 / \kappa}=$ $\xi h^{-1 / \kappa}$, we get

$$
\begin{aligned}
N(z(t)) & =e^{s} N\left(x\left(e^{\kappa s} t\right)\right) \\
& \leq e^{s} \beta_{0}\left(N\left(x_{0}\right), e^{\kappa s} t\right) \\
& \leq e^{s} \beta_{0}\left(e^{-s} N\left(z_{0}\right), e^{\kappa s} t\right) \\
& \leq \beta_{s}\left(N\left(z_{0}\right), t\right)
\end{aligned}
$$

with $\beta_{s}(r, t)=e^{s} \beta_{0}\left(e^{-s} r, e^{\kappa s} t\right) \in \mathcal{K} \mathcal{L}$, and finally

$$
N\left(z_{0}\right) \leq \xi h^{-1 / \kappa} \Rightarrow N(z(t)) \leq \beta_{s}\left(N\left(z_{0}\right), t\right)
$$

for all $t \geq 0$. In the case $\kappa<0$, a similar method can be used, based on the following recast of the announced result:

$$
N(x(t)) \leq \beta_{0}\left(N\left(x_{0}\right), t\right)+\xi h^{-1 / \kappa}, \quad \forall t \geq 0
$$

with $\beta_{0} \in \mathcal{K} \mathcal{L}$.

Step II. We shall now base the sequel of this proof on a rewriting of the system (5). Noting that $u\left(x\left(t_{k}\right)\right)=$ $u\left(x(t)+\left[x\left(t_{k}\right)-x(t)\right]\right)$, we define $f(x, z)=F(x, u(x+$ $z)$ ) and hence we see that the system (5) can be rewritten under the equivalent form

$$
\left\{\begin{array}{l}
\dot{x}=f(x, \Delta) \\
\Delta(t)=x\left(t_{k}\right)-x(t), \quad t \in\left[t_{k}, t_{k+1}\right)
\end{array}\right.
$$

The signal $\Delta$ is piecewise continuous and verifies $\dot{\Delta}=$ $-f(x, \Delta)$ and $\Delta\left(t_{k}\right)=0$. It is straightforward to check that, under Assumption 11, the hypotheses of Lemma 13 hold. Since here $x(t)$ and $\Delta(t)$ belong to the same space, namely $\mathbb{R}^{n}$, and $\nu(x)=\tilde{\nu}(x)=A x$ we can always select the homogeneous norm $\tilde{N}$ of Equation (7) to be equal to $N$. The origin being a globally asymptotically stable equilibrium of the $\nu$-homogeneous system $\dot{x}=f(x, 0)$ of degree $\kappa$, by virtue of [33, Theorem 2] there exists a 
$\nu$-homogeneous smooth Lyapunov function $V$ of degree $\mu>0$ with $\mu+\kappa>0$. Let us define $N(x)=V(x)^{1 / \mu}$. We will prove the theorem for this particular homogeneous norm $N$, but Proposition 10 implies that the theorem then holds for all homogeneous norms.

Step III. Step II and Lemma 13 lead to

$$
N(x(t)) \leq \max \{\beta(N(x(0)), t) ; C \underset{\tau \in[0, t]}{\operatorname{ess} \sup } N(\Delta(\tau))\}
$$

with $C>0$ and $\beta \in \mathcal{K} \mathcal{L}$ of Lemma 13 . We define $\mathbb{S}=$ $\left\{z \in \mathbb{R}^{n}: N(z)=1\right\}$ and for any $\varepsilon>0$

$\alpha(\varepsilon)=\sup \left\{\left\|d_{z} V F(y-z, u(y))\right\|: V(z) \leq \varepsilon^{\mu}\right.$ and $\left.y \in \mathbb{S}\right\}$

and $H=\sup _{0<\varepsilon<1 / C} \frac{\varepsilon^{\mu}}{\alpha(\varepsilon)} \in(0,+\infty]$. Let us mention that for any finite $\varepsilon>0, \alpha(\varepsilon)$ is finite, being the supremum of a continuous function on a compact set. Fix $h \in(0, H)$. There exists $\varepsilon \in(0,1 / C)$ such that $h \leq \frac{\varepsilon^{\mu}}{\alpha(\varepsilon)}$. With this couple $(h, \varepsilon)$ we shall now prove the following fact.

Fact. Consider $k$ such that $N\left(x\left(t_{k}\right)\right)^{-\kappa} \geq \frac{\alpha(\varepsilon) h}{\varepsilon^{\mu}}$, then we have

$$
N(x(t)) \leq \max \left\{\beta\left(N\left(x\left(t_{k}\right)\right), t-t_{k}\right) ; C \varepsilon N\left(x\left(t_{k}\right)\right)\right\}
$$

for all $t \in\left[t_{k}, t_{k+1}\right]$.

Remark first that if $x\left(t_{k}\right)=0$, then the asymptotic stability of the origin for the closed-loop system $\dot{x}=$ $F(x, u(x))$ implies that $x(t)=0$ for all $t \in\left[t_{k}, t_{k+1}\right]$ and the fact is proved. Now, to prove the fact when $x\left(t_{k}\right) \neq 0$, let us study the variations of $\Delta(t)$. We denote $s=\ln \left(N\left(x\left(t_{k}\right)\right)\right)$. Consider

$$
\begin{aligned}
t^{*} & =\inf \left\{t \geq t_{k}: N(\Delta(t))>\varepsilon N\left(x\left(t_{k}\right)\right)\right\} \\
& =\inf \left\{t \geq t_{k}: V(\Delta(t))>\varepsilon^{\mu} V\left(x\left(t_{k}\right)\right)\right\}
\end{aligned}
$$

Thus on the interval $\left[t_{k}, t^{*}\right]$ we have $V(\Delta(t)) \leq$ $\varepsilon^{\mu} V\left(x\left(t_{k}\right)\right)$, which implies $V(\exp (-A s) \Delta(t)) \leq \varepsilon^{\mu}$ and $\exp (-A s) x\left(t_{k}\right) \in \mathbb{S}$ given that $N\left(x\left(t_{k}\right)\right)=e^{s}$. We have

$$
\begin{aligned}
V\left(\Delta\left(t^{*}\right)\right)= & \int_{t_{k}}^{t^{*}} d_{\Delta(t)} V f\left(x(t), u\left(x\left(t_{k}\right)\right)\right) d t \\
\leq & \left(t^{*}-t_{k}\right) \sup _{t \in\left[t_{k}, t^{*}\right]}\left\|d_{\Delta(t)} V F\left(x(t), u\left(x\left(t_{k}\right)\right)\right)\right\| \\
\leq & \left(t^{*}-t_{k}\right) \sup \left\{\left\|d_{\tilde{z}} V F(\tilde{y}-\tilde{z}, u(\tilde{y}))\right\|:\right. \\
& \left.V(\exp (-A s) \tilde{z}) \leq \varepsilon^{\mu} \text { and } \exp (-A s) \tilde{y} \in \mathbb{S}\right\}
\end{aligned}
$$

and from Assumption 11 and Proposition 5, it follows that

$$
\begin{aligned}
V\left(\Delta\left(t^{*}\right)\right) \leq & e^{(\kappa+\mu) s}\left(t^{*}-t_{k}\right) \sup \left\{\left\|d_{z} V F(y-z, u(y))\right\|:\right. \\
& \left.V(z) \leq \varepsilon^{\mu} \text { and } y \in \mathbb{S}\right\} \\
\leq & N\left(x\left(t_{k}\right)\right)^{\kappa+\mu}\left(t^{*}-t_{k}\right) \alpha(\varepsilon)
\end{aligned}
$$

By continuity, we have $V\left(\Delta\left(t^{*}\right)\right)=\varepsilon^{\mu} V\left(x\left(t_{k}\right)\right)=$ $\varepsilon^{\mu} N\left(x\left(t_{k}\right)\right)^{\mu}$ which leads to

$$
\varepsilon^{\mu} N\left(x\left(t_{k}\right)\right)^{\mu} \leq N\left(x\left(t_{k}\right)\right)^{\kappa+\mu}\left(t^{*}-t_{k}\right) \alpha(\varepsilon)
$$

and then $t^{*}-t_{k} \geq \frac{\varepsilon^{\mu} N\left(x\left(t_{k}\right)\right)^{-\kappa}}{\alpha(\varepsilon)} \geq h$ that is $t^{*} \geq t_{k+1}$. Thus, for all $t \in\left[t_{k}, t_{k+1}\right]$ we have $N(\Delta(t)) \leq \varepsilon N\left(x\left(t_{k}\right)\right)$. Finally, inserting this inequality into (10) yields (11) and the fact is proved.

We shall now split the proof into three cases, whether $\kappa>0$ or $\kappa=0$ or $\kappa<0$.

Step IV: $\kappa>0$

The condition $N\left(x\left(t_{k}\right)\right)^{-\kappa} \geq \frac{\alpha(\varepsilon) h}{\varepsilon^{\mu}}$ is equivalent to $N\left(x\left(t_{k}\right)\right) \leq\left(\frac{\varepsilon^{\mu}}{\alpha(\varepsilon) h}\right)^{1 / \kappa}$. Let us denote

$$
B(h)=\left\{z \in \mathbb{R}^{n}: N(z) \leq\left(\frac{\varepsilon^{\mu}}{\alpha(\varepsilon) h}\right)^{1 / \kappa}\right\} .
$$

The set $B(h)$ is a compact neighborhood of the origin. If $x_{0} \in B(h),(11)$ leads to $N(x(t)) \leq N\left(x\left(t_{k}\right)\right)$ for all $k \in$ $\mathbb{N}$ and all $t \in\left(t_{k}, t_{k+1}\right]$. Therefore the system is stable and $N(x(t))$ converges to a limit $\ell \geq 0$ when $t \rightarrow+\infty$. Now, by using (11), we obtain for all $t \in\left[t_{k}, t_{k+1}\right]$ and $k \in \mathbb{N}$

$$
\begin{aligned}
N\left(x\left(t_{k+1}\right)\right) & \leq \max \left\{\beta\left(N\left(x\left(t_{k}\right)\right), t_{k+1}-t_{k}\right) ; C \varepsilon N\left(x\left(t_{k}\right)\right)\right\} \\
& \leq \max \left\{\beta\left(N\left(x\left(t_{k}\right)\right), \eta\right) ; C \varepsilon N\left(x\left(t_{k}\right)\right)\right\} .
\end{aligned}
$$

Following (8), we have $\beta(r, t)=r \beta\left(1, t r^{\kappa / \mu}\right)$ therefore the limit $\ell$ satisfies $\ell \leq \max \left\{\ell \beta\left(1, \eta \ell^{\kappa}\right) ; C \varepsilon \ell\right\} \leq \ell$. Thus $\ell=\max \left\{\ell \beta\left(1, \eta \ell^{\kappa / \mu}\right) ; C \varepsilon \ell\right\}=\ell \beta\left(1, \eta \ell^{\kappa / \mu}\right)$ since $C \varepsilon<1, \kappa>0$ and $\beta(r, 0)=r$. Hence $\ell=0$ or $\beta\left(1, \eta \ell^{\kappa / \mu}\right)=1$, but this second condition is also equivalent to $\ell=0$. The local asymptotic stability follows with $B(h)$ a subset of the domain of attraction.

Step $V: \kappa=0$

The condition $N\left(x\left(t_{k}\right)\right)^{-\kappa} \geq \frac{\alpha(\varepsilon) h}{\varepsilon^{\mu}}$ is equivalent to $h \leq$ $\frac{\varepsilon^{\mu}}{\alpha(\varepsilon)}$ and therefore always holds given the choice we have made for $\varepsilon$ in Step III. By similar arguments to the case $\kappa>0$, we obtain that, if $h<H$, the origin is a globally asymptotically stable equilibrium of the system (5).

Step VI: $\kappa<0$

The condition $N\left(x\left(t_{k}\right)\right)^{-\kappa} \geq \frac{\alpha(\varepsilon) h}{\varepsilon^{\mu}}$ is equivalent to $N\left(x\left(t_{k}\right)\right) \geq\left(\frac{\alpha(\varepsilon) h}{\varepsilon^{\mu}}\right)^{-\frac{1}{\kappa}}=\rho$. Hence $N\left(x\left(t_{k}\right)\right) \geq \rho$ ensures that $N\left(x\left(t_{k+1}\right)\right) \leq N(x(t))<N\left(x\left(t_{k}\right)\right)$ for all $t \in\left(t_{k}, t_{k+1}\right]$. However, the set $\left\{x \in \mathbb{R}^{n}: N(x)<\rho\right\}$ is not necessarily positively invariant. We will therefore need another analysis to prove that, starting with 
$N\left(x\left(t_{k}\right)\right)<\rho$, a trajectory cannot go "too far" from the origin. Let us define, for $\omega \geq 0$

$\theta(\omega)=\sup \left\{\left\|d_{z} V F(z, u(y))\right\|: V(z) \leq 1+\omega, V(y) \leq 1\right\}$.

Denoting $s=\frac{\ln (1+\omega)}{\mu}$, we get

$$
\begin{aligned}
\theta(\omega)= & \sup \left\{\left\|d_{z} V F(z, u(y))\right\|: V(\exp (-A s) z) \leq 1\right. \text { and } \\
& V(\exp (-A s) y) \leq 1 /(1+\omega)\} \\
= & \sup \left\{\left\|d_{\exp (A s) \tilde{z}} V F(\exp (A s) \tilde{z}, u(\exp (A s) \tilde{y}))\right\|:\right. \\
& V(\tilde{z}) \leq 1 \text { and } V(\tilde{y}) \leq 1 /(1+\omega)\}
\end{aligned}
$$

and from Assumption 11 and Proposition 5, it follows that

$$
\begin{aligned}
\theta(\omega)= & e^{(\kappa+\mu) s} \sup \left\{\left\|d_{\tilde{z}} V F(\tilde{z}, u(\tilde{y}))\right\|: V(\tilde{z}) \leq 1\right. \text { and } \\
& V(\tilde{y}) \leq 1 /(1+\omega)\} \\
= & (1+\omega)^{\frac{\kappa+\mu}{\mu}} \sup \left\{\left\|d_{\tilde{z}} V F(\tilde{z}, u(\tilde{y}))\right\|: V(\tilde{z}) \leq 1\right. \text { and } \\
& V(\tilde{y}) \leq 1 /(1+\omega)\} .
\end{aligned}
$$

Hence when $\omega \rightarrow+\infty$ we have $\theta(\omega) \sim \Omega \omega^{\frac{\kappa+\mu}{\mu}}$, with $\Omega=\sup \left\{\left\|d_{\tilde{z}} V F(\tilde{z}, u(0))\right\|: V(\tilde{z}) \leq 1\right\}$ and therefore $\frac{\omega}{\theta(\omega)} \rightarrow+\infty$, given that $\frac{\kappa+\mu}{\mu}<1$ since $\kappa<0$. Since the function $\omega \mapsto \frac{\omega}{\theta(\omega)}$ vanishes at $\omega=0$, there exists $\omega_{0}>0$ such that $\frac{\omega_{0}}{\theta\left(\omega_{0}\right)}=\frac{\varepsilon^{\mu}}{\alpha(\varepsilon)}$. Assume that $N\left(x\left(t_{k}\right)\right)<\rho$, i.e. $V\left(x\left(t_{k}\right)\right)<\rho^{\mu}$. Let us denote $t^{*}=\inf \left\{t \geq t_{k}: V(x(t)) \geq\left(1+\omega_{0}\right) \rho^{\mu}\right\}>t_{k}$. Hence for all $t \in\left[t_{k}, t^{*}\right]$ we have $V(x(t)) \leq\left(1+\omega_{0}\right) \rho^{\mu}$. Therefore, we obtain

$$
\begin{aligned}
V\left(x\left(t^{*}\right)\right)= & V\left(x\left(t_{k}\right)\right)+\int_{t_{k}}^{t^{*}} \frac{d}{d t} V(x(t)) d t \\
\leq & \rho^{\mu}+\left(t^{*}-t_{k}\right) \sup \left\{\left\|d_{x(t)} V F\left(x(t), u\left(x\left(t_{k}\right)\right)\right)\right\|:\right. \\
& \left.t \in\left[t_{k}, t^{*}\right]\right\} \\
\leq & \rho^{\mu}+\left(t^{*}-t_{k}\right) \sup \left\{\left\|d_{z} V F(z, u(y))\right\|:\right. \\
& \left.V(z) \leq\left(1+\omega_{0}\right) \rho^{\mu} \text { and } V(y) \leq \rho^{\mu}\right\} \\
\leq & \rho^{\mu}+\left(t^{*}-t_{k}\right) \rho^{\kappa+\mu} \theta\left(\omega_{0}\right) .
\end{aligned}
$$

Also, by continuity, $V\left(x\left(t^{*}\right)\right)=\left(1+\omega_{0}\right) \rho^{\mu}$ and therefore after simplification and using $\rho=\left(\frac{\alpha(\varepsilon) h}{\varepsilon^{\mu}}\right)^{-\frac{1}{\kappa}}$ we get $\omega_{0} \leq\left(t^{*}-t_{k}\right)\left(\frac{\alpha(\varepsilon) h}{\varepsilon^{\mu}}\right)^{-1} \theta\left(\omega_{0}\right)$ and given that $\frac{\omega_{0}}{\theta\left(\omega_{0}\right)}=$ $\frac{\varepsilon^{\mu}}{\alpha(\varepsilon)}$ we find $t^{*}-t_{k} \geq h$. Finally, $t^{*} \geq t_{k+1}$ and then for all $t \in\left[t_{k}, t_{k+1}\right]$ we have $V(x(t)) \leq\left(1+\omega_{0}\right) \rho^{\mu}$, which implies $N(x(t)) \leq\left(1+\omega_{0}\right)^{1 / \mu} \rho$.

Let us summarize. We have three cases:

- if $N\left(x\left(t_{k}\right)\right)<\rho$ then we have $N(x(t)) \leq\left(1+\omega_{0}\right)^{1 / \mu} \rho$ for all $t \in\left[t_{k}, t_{k+1}\right]$;

- if $\rho \leq N\left(x\left(t_{k}\right)\right) \leq\left(1+\omega_{0}\right)^{1 / \mu} \rho$ then we still have $N(x(t)) \leq\left(1+\omega_{0}\right)^{1 / \mu} \rho$ for all $t \in\left[t_{k}, t_{k+1}\right]$;
- if $N\left(x\left(t_{k}\right)\right) \geq\left(1+\omega_{0}\right)^{1 / \mu} \rho$ then $N\left(x\left(t_{k}\right)\right)^{-\kappa} \geq \frac{\alpha(\varepsilon) h}{\varepsilon^{\mu}}$ and by the Fact of Step III we have

$$
N(x(t)) \leq \max \left\{\beta\left(N\left(x\left(t_{k}\right)\right), t-t_{k}\right) ; C \varepsilon N\left(x\left(t_{k}\right)\right)\right\}
$$

for all $t \in\left[t_{k}, t_{k+1}\right]$.

We get from the first two points that the set $\{N(x) \leq$ $\left.\left(1+\omega_{0}\right)^{1 / \mu} \rho\right\}$ is positively invariant. Hence, if $N\left(x_{0}\right)>$ $\left(1+\omega_{0}\right)^{1 / \mu} \rho$, denoting $k^{*}=\max _{k \geq 0}\left\{N\left(x\left(t_{k}\right)\right) \geq(1+\right.$ $\left.\left.\omega_{0}\right)^{1 / \mu} \rho\right\}$, for all $0 \leq k \leq k^{*}$, we have $N\left(x\left(t_{k}\right)\right) \geq$ $\left(1+\omega_{0}\right)^{1 / \mu} \rho$. Therefore the third point gives us that $N\left(x\left(t_{k+1}\right)\right) \leq N\left(x\left(t_{k}\right)\right)$ for all $0 \leq k \leq k^{*}$ and thus $N\left(x\left(t_{k}\right)\right)<\bar{N}\left(x_{0}\right)$ for all $0<k \leq \bar{k}^{*}$. Using again the shape of the function $\beta$, for $0 \leq k<k^{*}$ and $t \in\left[t_{k}, t_{k+1}\right]$ we get

$$
\begin{aligned}
N(x(t)) & \leq \max \left\{\beta\left(N\left(x\left(t_{k}\right)\right), \eta\right) ; C \varepsilon N\left(x\left(t_{k}\right)\right)\right\} \\
& \leq N\left(x\left(t_{k}\right)\right) \max \left\{\beta\left(1, \eta N\left(x\left(t_{k}\right)\right)^{\kappa / \mu}\right) ; C \varepsilon\right\} \\
& \leq N\left(x\left(t_{k}\right)\right) \max \left\{\beta\left(1, \eta N\left(x_{0}\right)^{\kappa / \mu}\right) ; C \varepsilon\right\} \\
& \leq N\left(x\left(t_{k}\right)\right) q
\end{aligned}
$$

where $q=\max \left\{\beta\left(1, \eta N\left(x_{0}\right)^{\kappa / \mu}\right) ; C \varepsilon\right\}<1$, given that $\beta \in \mathcal{K} \mathcal{L}$ and $\beta(r, 0)=r$. Therefore

$$
N(x(t)) \leq \max \left\{N\left(x_{0}\right) q^{k} ;\left(1+\omega_{0}\right)^{1 / \mu} \rho\right\}, t \in\left[t_{k}, t_{k+1}\right]
$$

and we get the global asymptotical stability of the set $\left\{x \in \mathbb{R}^{n}: N(x) \leq\left(1+\omega_{0}\right)^{1 / \mu} \rho\right\}=\left\{x \in \mathbb{R}^{n}: N(x) \leq\right.$ $\left.\xi h^{-1 / \kappa}\right\}$.

Remark 14 With the notations of Theorem 12, in the case $\kappa<0$, the conclusion is equivalent to

$$
N(x(t)) \leq \beta_{0}(N(x(0)), t)+\xi h^{-1 / \kappa}, \quad \forall 0<h<H
$$

with $\beta_{0}$ a class $\mathcal{K} \mathcal{L}$ function, that is, the system (5) is ISS w.r.t. the input $h$ with asymptotic gain $\gamma(h)=\xi h^{-1 / \kappa}$. Consequently, the origin is globally practically stable, see [34] for details. Let us also remark that the linear case is included in the case $\kappa=0$.

The statement of Theorem 12 does not provide an explicit method for estimating the constants $H$ and $\xi$. From this perspective, it could be seen as a qualitative result on sampled-data systems. However, up to a constant, the Theorem 12 gives a precise estimation of the dependency on $h$ of the domain of attraction (when $\kappa>0$ ) and of the stable set (when $\kappa<0$ ), which is a quantitative data. Moreover, we would like to point out that, in most particular cases, the constants $H$ and $\xi$ can be estimated mathematically (if a Lyapunov function is known) or by computer simulations (see Section 4).

Even though Theorem 12 is written to emphasize the effects of the communication network on a system 
asymptotically stabilized by a state feedback, the result also applies to a system asymptotically stabilized by an observer-based controller, provided that this observer is homogeneous as well. We keep considering the system (3) verifying Assumption 11. We endow the system with an output $y=j(x)$. As beforehand, we assume known a state feedback $u(x)$ such that the origin is a globally asymptotically stable equilibrium for the closed-loop system. We assume that an observer is added to the system

$$
\dot{\hat{x}}=G(y, \hat{x})
$$

with $G$ continuous, in such a way that the origin of $\mathbb{R}^{n} \times$ $\mathbb{R}^{n}$ is a globally asymptotically stable equilibrium of the following system

$$
\left\{\begin{array}{l}
\dot{x}=F(x, u(\hat{x})) \\
\dot{\hat{x}}=G(j(x), \hat{x})
\end{array}\right.
$$

Now we want to study the impact of a sampling on the stability of this system. Indeed, the value of $\hat{x}$ will be updated only at the sampling instants $t_{k}$, leading to

$$
\left\{\begin{array}{l}
\dot{x}(t)=F\left(x(t), u\left(\hat{x}\left(t_{k}\right)\right)\right), \quad t \in\left[t_{k}, t_{k+1}\right) \\
\hat{x}\left(t_{k+1}\right)=\hat{x}\left(t_{k}\right)+\left(t_{k+1}-t_{k}\right) G\left(j\left(x\left(t_{k}\right)\right), \hat{x}\left(t_{k}\right)\right)
\end{array}\right.
$$

Proposition 15 Assume that the system (12), defined on $\mathbb{R}^{n} \times \mathbb{R}^{n}$, is $\nu$-homogeneous of degree $\kappa$ with $\nu(x, \hat{x})=$ $(A x, A \hat{x})$. Assume also that the sampled system (13) is such that the sampling times satisfy (4).

(1) If $\kappa>0$ then the origin is a locally asymptotically stable equilibrium of the system (13) and there exists $\xi>0$ such that the set $\left\{(x, \hat{x}) \in \mathbb{R}^{n} \times \mathbb{R}^{n}\right.$ : $\left.N(x)+N(\hat{x}) \leq \xi h^{-1 / \kappa}\right\}$ is a subset of the domain of attraction.

(2) If $\kappa=0$ then there exists $H>0$ such that if $h<H$ then the origin is a globally asymptotically stable equilibrium of the system (13).

(3) If $\kappa<0$ then there exists $\xi>0$ such that the set $\left\{(x, \hat{x}) \in \mathbb{R}^{n} \times \mathbb{R}^{n}: N(x)+N(\hat{x}) \leq \xi h^{-1 / \kappa}\right\}$ is globally asymptotically stable w.r.t. the system (13).

Proof. Let us denote

$$
\bar{F}(X, U)=\left(\begin{array}{c}
F\left(X_{1}, U_{1}\right) \\
U_{2}
\end{array}\right)
$$

for $X=\left(X_{1}, X_{2}\right) \in \mathbb{R}^{n} \times \mathbb{R}^{n}$ and $U=\left(U_{1}, U_{2}\right) \in \mathbb{R}^{n} \times$ $\mathbb{R}^{n}$ and consider

$$
\dot{X}=\bar{F}(X, U(X)) \text {. }
$$

If we apply to (14) the state feedback given by

$$
\left\{\begin{array}{l}
U_{1}(X)=u\left(X_{2}\right) \\
U_{2}(X)=G\left(j\left(X_{1}\right), X_{2}\right)
\end{array}\right.
$$

we find

$$
\left\{\begin{array}{l}
\dot{X}_{1}=F\left(X_{1}, u\left(X_{2}\right)\right) \\
\dot{X}_{2}=G\left(j\left(X_{1}\right), X_{2}\right)
\end{array}\right.
$$

which is (12), up to a renaming of the variables. Now, (14) with sampled input (15) becomes

$$
\left\{\begin{array}{l}
\dot{X}_{1}(t)=F\left(X_{1}(t), u\left(X_{2}\left(t_{k}\right)\right)\right) \\
\dot{X}_{2}(t)=G\left(j\left(X_{1}\left(t_{k}\right)\right), X_{2}\left(t_{k}\right)\right)
\end{array}\right.
$$

and the right hand side of the second line being constant, the second line can be integrated. It yields

$$
\left\{\begin{array}{l}
\dot{X}_{1}(t)=F\left(X_{1}(t), u\left(X_{2}\left(t_{k}\right)\right)\right) \\
X_{2}\left(t_{k+1}\right)=X_{2}\left(t_{k}\right)+\left(t_{k+1}-t_{k}\right) G\left(j\left(X_{1}\left(t_{k}\right)\right), X_{2}\left(t_{k}\right)\right)
\end{array}\right.
$$

which is (13), up to a renaming of the variables. Moreover, the Assumption 11 holds for $\bar{F}$. Therefore, we apply Theorem 12 to (14) which concludes the proof.

\section{The double integrator}

Let us consider the double integrator with a sampleddata input

$$
\dot{x}(t)=\left[\begin{array}{ll}
0 & 1 \\
0 & 0
\end{array}\right] x(t)+\left[\begin{array}{l}
0 \\
1
\end{array}\right] u\left(t_{k}\right), \quad t \in\left[t_{k}, t_{k+1}\right)
$$

which has been studied in [25] as a typical example and the control law

$$
u(x)=-k_{1}\left\lfloor x_{1}\right\rceil^{\alpha}-k_{2}\left\lfloor x_{2}\right\rceil^{\frac{2 \alpha}{1+\alpha}}
$$

with $\alpha>0$ and $k_{1}, k_{2}>0$. By using $V(x)=$ $\frac{k_{1}}{1+\alpha}\left|x_{1}\right|^{1+\alpha}+\frac{x_{2}^{2}}{2}$, we get $\dot{V}(x)=-k_{2}\left|x_{2}\right|^{\frac{1+3 \alpha}{1+\alpha}} \leq 0$. A straightforward application of the LaSalle's principle shows that the origin of the continuous closed-loop system (16)-(17) without the sampled-data input is a globally asymptotically stable equilibrium. In this example, we consider $\nu=A x$ with $A=\operatorname{Diag}\left(1, \frac{1+\alpha}{2}\right)$, we see that the closed-loop system (16)-(17) satisfies Assumption 11 with degree $\kappa=\frac{\alpha-1}{2}$. So, we can apply Theorem 12 . Moreover, we choose a constant sampling period

$$
t_{k}=k T, \quad T>0, k \in \mathbb{N}
$$

in order to use well known results about linear systems having a constant sampling period. For the simulations, we take $k_{1}=k_{2}=1$.

- If we select $\alpha=1$ then the closed-loop system (16)(17)-(18) is linear. In this case, we know that the linear closed-loop sampled-data system (16)-(17)-(18) is asymptotically stable if and only if the matrix $\Lambda(T)$ of the linear difference equation associated with (16)(17) and defined in $[6,35]$ is Schur. For the double integrator, $\Lambda(T)$ is Schur if and only if $T<T_{\text {Schur }}=2 \mathrm{~s}$. 
- If we select $\alpha>1$ then the closed-loop system (16)(17)-(18) is homogeneous of positive degree. Due to Theorem 12, the local asymptotic stability pertains even if we have $T>T_{\text {Schur }}=2 \mathrm{~s}$. We plot in simulation the shape of the domains of attraction for $\alpha=8$ and different values of $T$ on Figure 1 and the shape of the domains of attraction for $T=6 \mathrm{~s}$ and different values of $\alpha$ on Figure 2 .

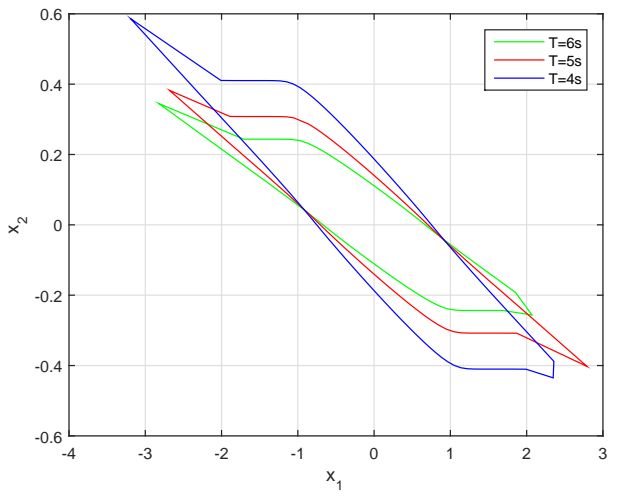

Fig. 1. Domains of attraction for $\alpha=8$

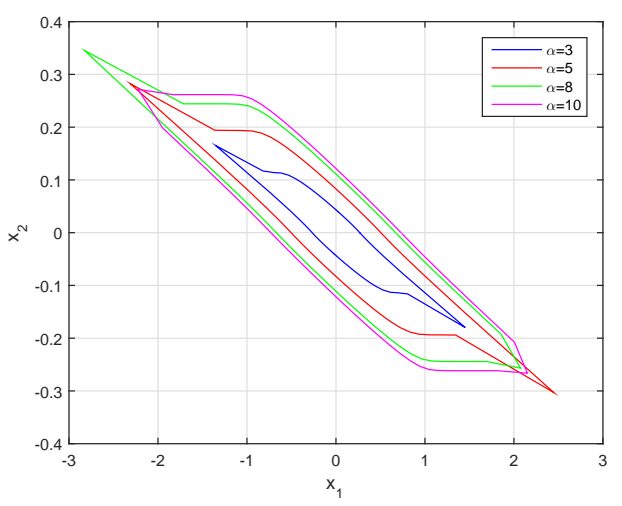

Fig. 2. Domains of attraction for $T=6 \mathrm{~s}$

- If we select $\alpha<1$ then the closed-loop system (16)(17)-(18) is homogeneous of negative degree. Due to Theorem 12, the global asymptotic set stability pertains even if $T>T_{\text {Schur }}=2 \mathrm{~s}$. We plot in simulation the asymptotically stable sets for $\alpha=0.1$ and $T=4 \mathrm{~s}$, $T=5 \mathrm{~s}$ and $T=6 \mathrm{~s}$ on Figure 3 .

The case of the $n$-integrator can be treated in the same way by using for instance the controllers given in $[18,31]$.

\section{Conclusion}

In this article, an emulation approach based on inputto-state stability properties of systems satisfying an homogeneous condition is developed for the stability of nonlinear systems with sampled-data inputs. The main

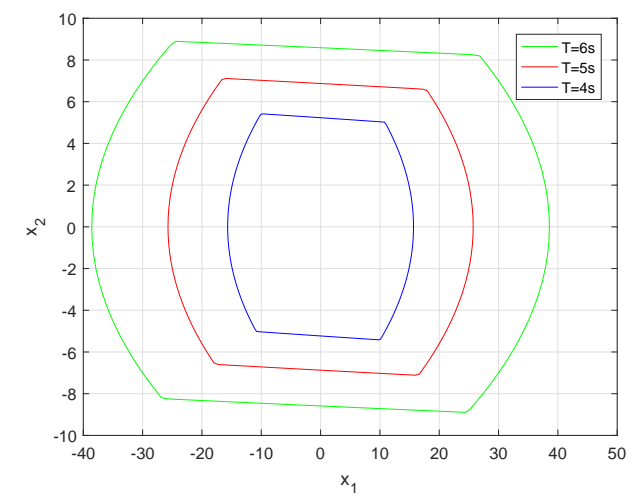

Fig. 3. Asymptotically stable sets for several $T$

result shows that if it is possible to build a stabilizing feedback control for a continuous system such that the closed-loop system with sampled-data inputs satisfies the homogeneity property, then it is possible to preserve different notions of stability that depend on the degree $\kappa$ for the closed-loop system with aperiodic sampled-data inputs. Its usefulness is highlighted with the case of the double integrator.

\section{References}

[1] W. Zhang, M. S. Branicky, S. M. Phillips, Stability of networked control systems, IEEE Control Systems 21 (1) (2001) 84-99.

[2] A. Bemporad, M. Heemels, M. Johansson, Networked control systems, Vol. 406 of Lecture Notes in Control and Information Sciences, Springer, 2010.

[3] F.-Y. Wang, D. Liu, Networked control systems, Springer, 2008.

[4] G. C. Walsh, H. Ye, L. G. Bushnell, Stability analysis of networked control systems, IEEE Transactions on Control Systems Technology 10 (3) (2002) 438-446.

[5] K. Liu, E. Fridman, L. Hetel, Networked control systems: A time-delay approach, in: European Control Conference, Strasbourg, France, 2014, pp. 1434-1439.

[6] L. Hetel, C. Fiter, H. Omran, A. Seuret, E. Fridman, J.-P. Richard, S. I. Niculescu, Recent developments on the stability of systems with aperiodic sampling: an overview, Automatica 76 (2017) 309-335.

[7] D. Nešić, A. R. Teel, Input-output stability properties of networked control systems, IEEE Transactions on Automatic Control 49 (10) (2004) 1650-1667.

[8] H. Omran, L. Hetel, J.-P. Richard, F. Lamnabhi-Lagarrigue, On the stability of input-affine nonlinear systems with sampled-data control, in: European Control Conference, Zürich, Switzerland, 2013, pp. 2585-2590.

[9] N. W. Bauer, P. J. H. Maas, W. P. M. H. Heemels, Stability analysis of networked control systems: A sum of squares approach, Automatica 48 (8) (2012) 1514-1524.

[10] M. C. F. Donkers, W. P. M. H. Heemels, N. Van De Wouw, L. Hetel, Stability analysis of networked control systems using a switched linear systems approach, IEEE Transactions on Automatic Control 56 (9) (2011) 2101-2115. 
[11] D. Nešić, A. R. Teel, A framework for stabilization of nonlinear sampled-data systems based on their approximate discrete-time models, IEEE Transactions on automatic control 49 (7) (2004) 1103-1122.

[12] H. Fujioka, Stability analysis of systems with aperiodic sample-and-hold devices, Automatica 45 (3) (2009) 771-775.

[13] F. Mazenc, M. Malisoff, T. N. Dinh, Robustness of nonlinear systems with respect to delay and sampling of the controls, Automatica 49 (6) (2013) 1925-1931.

[14] A. Seuret, A novel stability analysis of linear systems under asynchronous samplings, Automatica 48 (1) (2012) 177-182.

[15] D. Nešić, A. R. Teel, D. Carnevale, Explicit computation of the sampling period in emulation of controllers for nonlinear sampled-data systems, IEEE Transactions on Automatic Control 54 (3) (2009) 619-624.

[16] V. Zubov, On ordinary differential equations with generalized homogeneous right-hand sides (in Russian), Izvestiya vuzov, Matematika 1 (2) (1958) 80-88.

[17] L. P. Rothschild, E. M. Stein, Hypoelliptic differential operators and nilpotent groups, Acta Mathematica 137 (1) (1976) 247-320.

[18] S. P. Bhat, D. S. Bernstein, Geometric homogeneity with applications to finite-time stability, Mathematics of Control, Signals and Systems 17 (2) (2005) 101-127.

[19] M. Kawski, Geometric homogeneity and stabilization, in: Nonlinear Control Systems Design, Elsevier, 1995, pp. 147152 .

[20] E. Moulay, Stability and stabilization of homogeneous systems depending on a parameter, IEEE Transactions on Automatic Control 54 (6) (2009) 1382-1385.

[21] A. Anta, P. Tabuada, To sample or not to sample: Selftriggered control for nonlinear systems, IEEE Transactions on Automatic Control 55 (9) (2010) 2030-2042.

[22] D. S. Laila, D. Nešić, A. R. Teel, Open-and closedloop dissipation inequalities under sampling and controller emulation, European Journal of Control 8 (2) (2002) 109125 .

[23] D. Nešić, A. R. Teel, E. D. Sontag, Formulas relating $\mathcal{K} \mathcal{L}$ stability estimates of discrete-time and sampled-data nonlinear systems, Systems \& Control Letters 38 (1) (1999) 49-60.

[24] Y. S. Suh, Stability and stabilization of nonuniform sampling systems, Automatica 44 (12) (2008) 3222-3226.

[25] E. Bernuau, W. Perruquetti, D. Efimov, E. Moulay, Robust finite-time output feedback stabilisation of the double integrator, International Journal of Control 88 (3) (2015) 451-460.

[26] S. P. Bhat, D. S. Bernstein, et al., Continuous finitetime stabilization of the translational and rotational double integrators, IEEE Transactions on Automatic Control 43 (5) (1998) 678-682.

[27] N. P. Bhatia, G. P. Szegö, Stability theory of dynamical systems, Vol. 161 of Classics in Mathematics, Springer, 2002.

[28] E. D. Sontag, Y. Wang, On characterizations of the inputto-state stability property, Systems \& Control Letters 24 (5) (1995) 351-359.

[29] G. Teschl, Ordinary Differential Equations and Dynamical Systems, Vol. 140 of Graduate Studies in Mathematics, Amer. Math. Soc., Providence, 2012.

[30] A. Bacciotti, L. Rosier, Liapunov functions and stability in control theory, 2nd Edition, Communications and Control Engineering, Springer, 2005.
[31] Y. Hong, Finite-time stabilization and stabilizability of a class of controllable systems, Systems \& Control Letters 46 (4) (2002) 231-236.

[32] E. Bernuau, D. Efimov, W. Perruquetti, On the robustness of homogeneous systems and a homogeneous small gain theorem, in: IEEE Conference on Control Applications, Antibes, France, 2014, pp. 929-934.

[33] L. Rosier, Homogeneous lyapunov function for homogeneous continuous vector field, Systems \& Control Letters 19 (6) (1992) 467-473.

[34] A. R. Teel, J. Peuteman, D. Aeyels, Semi-global practical asymptotic stability and averaging, Systems \& control letters 37 (5) (1999) 329-334.

[35] S. Maalej, C. Fiter, L. Hetel, J.-P. Richard, State-dependent sampling for linear time invariant systems: a discrete time analysis, in: 20th IEEE Mediterranean Conference on Control \& Automation, 2012, pp. 1129-1134. 